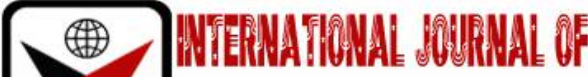

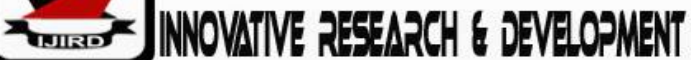

ISSN 2278-0211 (Online)

\section{Internet Addiction, Neuroplasticity and Education}

\author{
Dr. Madu, Virginia Nnenna \\ Chief Lecturer, Department of Educational Psychology \\ Federal College of Education, Obudu, Nigeria
}

\begin{abstract}
:
The benefit of information and communication technology is enormous, yet not without its side effects. The lapses arise more from misuse or overuse of internet-based activities. The terms used to describe this practice include, but not exhaustive the following, internet addiction, problematic internet use, internet overuse and maladaptive internet use. This paper explored the factors predisposing people to internet addiction, the interplay of internet addiction and neuroplasticity, physiological, psychological and social impact of internet addiction. It went further to highlight the effects of internet addiction on learning and advances educational interventions for curbing internet addiction. Some of the approaches include cognitive behavioural approaches, purposeful creation of new neural partway based on creativity and problem solving and tapping from positive psychology exercises.
\end{abstract}

Keywords: Neuroplasticity, Depression and Neurological Complications

\section{Introduction}

The internet which provides a variety of information and communication facilities using standardised communication protocols enables people to connect to virtual world for various purposes such as recreational, academic, social, business and leisure time activities. However, the abuse, misuse or overuse of internet has attracted the attention of Psychologists of various fields including education. The terms used to describe this scenario include the following; internet addiction, problematic internet use, problematic computer use, internet overuse, maladaptive internet use, pathologic internet use etc. For this work, addiction to internet is used to denote preoccupation or compulsive engagement to internet activities despite adverse consequences. Just as addiction to drug can bring about brain changes that can lead to mental health issues such as depression, anxiety, schizophrenia to mention but a few, addiction to internet can equally alter the human brain in structure and function. This is because the human brain is plastic, and can rewire itself as it is constantly exposed to a new experience.

The ability of the human brain to rewire itself otherwise known as neuroplasticity is something great, for that is the basis for learning and other positive changes. However, the same process can cause harm to an individual as a result of uncontrolled exposure or compulsive use of internet. Currently internet addiction is being seriously considered as a disorder, and that goes to show how serious the attendant problems are. Internet addiction can lead to a host of problems such as neurological complications, psychological and social problems, all of which have educational implications. This paper is addressed under the following sub-headings:

- Factors predisposing people to internet addiction

- The interplay of internet addiction and neuroplasticity

- Physiological, psychological and social impact of internet addiction

- Effect of internet addiction on learning

- Educational interventions for internet addiction

- Summary and conclusions

\section{Factors Predisposing People to Internet Addiction}

A number of factors could make an individual susceptible to internet addiction. These factors range from the kind of activities one engages in, to the apps that are of interest to an individual, personality factors, certain health and psychological conditions and owning the necessary device. Some research findings will throw light on these points.

In a study conducted by Prabhakaran, Patel, Ganjiwale and Somashekhar (2016), which investigated factors associated with internet addiction among school going adolescents in Vadodara, online friendship was found to be a significant predictor of internet addiction. Also, psychological factors such as using internet to escape from selfdissatisfaction, to deal with stress, emotional regulation along with individual temperaments have been found to be associated with the development of internet addiction and high-risk behaviours among adolescents (Prabhakaran et al, 2016). This same study found that owning a personal device, use of smart phones, use of internet for charting, making online friendship, online shopping, watching movies, online gaming was significantly associated with IA. The study also revealed that social networking sites such as Facebook, twitter, Instagram and instant messaging apps promote IA. 
Kuss, Griffiths and Binder (2013) investigated the prevalence and risk factors in internet addiction among students and came up with a result which showed that a combination of online gaming and openness to experience increased the risk of internet addiction. In additions, frequent usage of online shopping, social online activities, high neuroticism, and low agreeableness significantly increased the chances of being addicted to the internet. Another study by $\mathrm{Wu}$, Lee, Liao and Chang (2015) which investigated risk factors of internet addiction among internet users showed that neuroticism, life impairment, and internet use time were the main predictors of internet addiction.

Psychological conditions such anxiety and depression as well as lack of emotional support can predispose individuals to internet addiction. Such conditions can cause individuals to turn to internet as a way of easing off stress and loneliness

\section{Interplay of Internet Addiction and Neuroplasticity}

The compulsion to continually engage in an activity or behaviour despite the negative impact on the person's ability to remain mentally and/or physically healthy and functional in the home and community is what defines behavioural addiction of which internet addiction is one. The person may find the activity rewarding psychologically or get "high" while engaged in the activity, but latter feels guilty, remorse or even overwhelmed by the consequences of that continued choice. The human brain has an enormous ability to reorganize itself by forming new connections between brain cells. Throughout life the human brain changes in response to new experiences. It becomes pertinent that one should carefully choose the kind of activities to engage in, and the extent of engagement. A student who spends her precious time in playing internet games to the extent of getting addicted to it is rewiring in the wrong direction, the same applies to internet dating, online friendship and the like. Such activities strengthen the neurons for ruminating, mind wondering and weakens neurons that should be used for creative and gainful activities.

\section{Physiological, Psychological and Social Impact of Internet Addiction}

Internet use on its own is not something bad as it has a number of advantages which can promote social life, academic excellence, business and careers generally. However, when we talk about internet addiction, like any other addiction, we focus on the negative. Here we consider the physiological, psychological and social impact of internet addiction on internet addicts.

Reed, Romano, Re, Roaro, Osborne, Vigaro, and Truzoh (2017) in a study; differential physiological changes following internet exposure in higher and lower problematic internet users, assessed one hundred and forty four participants for physiological (blood pressure and heart rate) and psychological,(mood and state anxiety) function before and after an internet session. Subjects also completed a psychometric examination relating to their usage of the internet as well as their levels of depression and state anxiety. Result showed that individuals who identified themselves as having PIU displayed increases in heart rate and systolic blood pressure as well as reduced mood and increased state anxiety following cessation of internet session. There were no such changes in individuals with no self-reported PIU. These changes were independent of levels of depression and state anxiety.

Internet addiction is also linked to functional brain changes in the prefrontal cortex, temporal cortex and ventral striatum and structural brain changes in parts of prefrontal cortex.

Internet addiction causes disruptions of functional connectivity and such disruptions might be linked to behavioural impairments (Wee, Zhao, Yap, Wu, Shi, Price, 2014). In addition, IAD alters the volume of the brain. The brain continues to negatively transform as long as the addiction continues. Tian, Chen, Zhang Du, Hou, Xhao and Zhang (2014) assessed brain dopamine D2 (D2)/ serotonin 2A (5-HT2A) receptor function and glucose metabolism in the same subjects by positron emission tomography (PET) imaging approach, and investigated whether correlation exists between D2 receptor and glucose metabolism. Result showed a significant decrease in glucose metabolism in the prefrontal, temporal and limbic systems. Dysregulation of D2 receptor was observed in the striatum, and was correlated to years of overuse. A low level of D2 receptors in the striatum was significantly associated with decreased glucose metabolism in the orbitofrontal cortex. This implies that IAD interferes with glucose in the brain system which can in turn affect the functioning of the brain and learning.

The world Health Organization (WHO) classifies cell phone radiation as a possible human carcinogen due to an increased risk of brain cancer from long-term use of cell phones. Even short-term use of cell phones is said to change brain activities in a way that is not yet clear.

Socially, internet generally impacts on the way individuals socialize and relate to other people. This is more on the side of internet addicts. Healthy social life activities are sacrificed at the expense of internet addiction. A person with IA may turn down invitation to attend an occasion simply because the person wants to be glued to internet. Inability to know when to stop is in itself a problem. To be obsessed in any activity is a social problem, the person spends more and more time on the net at the expense of meaningful activities that should have been attended to. It has been observed that people have lost their lives as a result of IAD which would not permit them to know when to stop and think; such as people moving on the road and operating their phones. In this way, people have run into moving vehicles and are crushed. Thus, creating pain for their loved ones.

\section{Impact of Internet Addiction on Learning}

Internet addiction can derail the focus and life of the students. It feeds students with myriads of information that can overwhelm them and make them vulnerable to reckless life. Time on internet can overtake time for study and meaningful school activities. 
IAD decreases creativity and problem solving. Students no longer exercise creative thinking in other to get their problems solved. There is overdependence on the net for every bit of task. Often the information gotten from the net is not processed for real knowledge and retention.

Internet addiction causes students to stay up late at night thus causing disruption in their body circadian rhythms which in turn result in all sorts of problems such as insufficient sleep, aggression, tiredness, fatigue, and trouble concentrating. If this continues unchecked, the individual may develop major depression. With such features the student will hardly study effectively, academic activities will suffer resulting perhaps in poor performance and social adjustment problems.

\section{Educational Interventions for Internet Addiction}

\subsection{Cognitive Approach}

Students need to be totally reoriented on some of the factors that predispose them to internet addiction and the harm associated with it. Considering the causal factors, students should be taught to make wise choices in the sites they visit and what they do in chosen sites given that these two factors account for IA. Students should as much as possible avoid online friendship, using internet to escape from depression, anxiety, loneliness etc. they should avoid online shopping, online gaming and watching videos online.

As much as possible, students should use internet only to solve real life problems such as for research purposes. Students should limit their use of social networking sites. One must not be in all the social networking sites such as Facebook, Instagram, Twitter, U-tube, and What Sapp.

\subsection{Cognitive Behavioural Approach}

Psychological conditions such as depression and anxiety should be managed using healthy approaches such as cognitive behavioural and not ruminating on social network sites. Real life relationships provide greater opportunity for verbal discussion which in itself is therapeutic. If a friend or family member must be communicated through phone, it is advisable to call the person instead of sending text messages, as this creates room for talking and laughing. Students should tap into brain plasticity to reinvent themselves and carve out better lives for themselves (Madu, 2019) by weakening engagement to internet addiction. To achieve this, students should be taught some of the basic principles upon which brain plasticity works, such as the fact that the human brain takes on the information you feed into it. On this principle, a student suffering from depression can have a depression free mind-set, by mindfully focussing on his brain and telling himself positive things as well as trying to act in that direction. Following the principle of neuroplasticity, recovery is not automatic, you need to be focused, dedicated and persistent. As an individual focus on noble things and act in that direction, new neural connections are formed, and the existing ones connected to depression weaken. As the individual gets used to the new ways of thinking and acting, the weakened neural partway eventually dies. As the saying goes, 'use it or lose it'. Students should regularly rest their minds on feelings of wellness, then the brain will gradually take the shape of confidence, strength and peace.

Part of the cognitive approach to address IA is to let the students know the harm they are doing to themselves through IA. The World Health Organization classifies cell phones radiation as a possible human carcinogen associated with the risk of brain cancer; to be aware of this outcome and continue in IA is suicidal.

\subsection{Purposeful Creation of New Neural Pathways}

For students who have sustained brain damage due to IA, such as the disruption of functional connectivity linked to behavioural impairment, such students should avail themselves of the opportunity of brain malleability and recreate neural pathways built on desirable habits such as creativity and problem solving. This can be achieved following the principles of neuroplasticity as aforementioned. The principle of neuroplasticity is not only used to address existing problems, it can be employed to learn a desirable habit that one lacks.

\subsection{Positive Psychology Interventions}

Educational Psychologists and Guidance Counsellors should make use of positive psychology in helping students with IA tendencies to reduce the compulsion. Positive psychology promises high level of satisfaction, positive feelings such as enjoyment, balance between the available skill and the task challenges. Students should be encouraged to engage on activities that increase positive emotions, and enhance social relationship of individuals. Encourage students to get engaged in team work and outdoor activities. Here are six exercises for positive emotions as given by Battey (2016) and elaborated by the present author.

- Journaling three blessings (Seligman, 2011 cited in Battey, 2016), according to the author, every day at the end of the day, write down three things, large or small that went well for you and why they went well. This exercise has been shown to improve symptoms of depression over a timeframe of a few months. Experiencing and savouring the moment is a powerful way of connecting with our inner selves in a positive way and as Madu (2018) puts it positivity begets positivity.

- Practicing mindfulness, example, mindful breathing, added to this, are mindful attention and mindful actions.

- $\quad$ Practice loving kindness meditation. Moments of love for self and others can increase health, vitality and wellbeing. We should create more of such experiences and reflect on them in our quiet times. 
- Reframing negative events. How we interpret events in the world around us has influence on our thought and emotions. We should develop skill to deal with adversity and become more resilient and positive, such as seeing the good in every bad situation.

- $\quad$ Creating positive experiences. This is similar to creating new neural part ways of choice. Positive experiences especially shared ones create lasting impact on our emotions

- Holding a good posture. There is a link between posture and emotions, just as it exists between thought and emotion. So, we should cautiously maintain a body posture that would portray positive emotions and confidence. The adjustment should begin with the inner man, as the spiritual controls the physical.

- Educational programmes designed to address internet addiction

Educational programmes can be designed for the purpose of addressing IA tendencies. Such educational programmes should cover conscious internet use, academic motivation, effective use of time, mindfulness focusing, and self-regulation.

Cigdem (2016), carried out an educational intervention aimed at reducing internet addiction tendencies, the intervention sought the effect of training programme developed to increase conscious internet use, academic motivation and effective use of time on internet addiction tendencies of adolescents in high school. The participants were 30 randomly selected students who completed the measuring scale for internet addiction. The internet addiction tendency was measured with Problematic Internet Use Scale (PIUS). The study was a pre-test post- test design. The study group received a five -session educational programme designed to increase conscious internet use, academic motivation and efficient use of time. The final test measurement was completed ten days after the end of the experimental procedure, with a follow up measurement completed 6 months after the end of the experimental procedure. Data analysis used two-way variance analysis for the split-plot. The result reveal that the educational programme developed to increase conscious internet use, academic motivation and efficient use of time is effective at reducing adolescent`s internet addiction tendencies.

Educational psychologists are therefore encouraged to design similar educational programmes for reducing internet addiction tendencies. Future educational programmes for such purposes may focus on mindfulness and selfregulation.

\section{Summary and Conclusion}

In summary, the awareness of the physiological, psychological and social impact of internet addiction and particularly its impact on learning should compel any sensible individual to be guided on how to come out of it or not to get involved. For the victims of internet addiction, they should avail themselves of the approaches proffered in this work to come out of it such as the cognitive behavioural approach, purposeful creation of new neural partway based on creativity and problem solving, making use of positive psychology and availing themselves of any educational programme designed to curb internet addiction. The suggestions are also helpful to non-internet addicts, so that they don`t become victims. All stake holders in child development should be alert to this disorder and strive for early intervention. Educational Psychologists and Guidance Counsellors should design educational programmes for the purpose of curbing internet addiction among students.

\section{References}

i. Battey, S. (2016). 6 exercises for positive emotions. https://positivepsychology.com> Cigdem, B. C. (2016). Educational intervention for reducing internet addiction tendencies. Doi:10.15805/addicta.2016.3.0021 3(3). https://addicta.com.tr>article>edu

ii. Kuss, D. J., Griffiths, M.D., \& Binder, J.F. (May, 2013). Internet addiction in students: prevalence and risk factors. https://www.academia.edu>kuss.... d....

iii. Madu, V.N. (2018). Locus of control, depressive symptoms and perceived academic achievement of Learners: a systemic review. Global Journal of Educational Research 7, 31-37. https://www.ajol. Info>article.

iv. Prabhakaran A.C.A., Patel, V.R., Ganjiwale, D.J., \& Somashekhar, M. (2016). Factors associated

v. With internet addiction among school going adolescents in Vadodara. Journal of family Med Prim Care, Oct-Dec, 5(4):765-769 doi 10.4103/2249-4868.20149

vi. $\quad$ Reed, P., Romano, M., Federica, R., Alexandra R., Lisa, A.O., Caterina, Vigaro \& Roberto, T. (2017). Differential physiological changes following internet exposure in higher and lower problematic internet users. https://www.ncbi.nlh.gov>pub

vii. Tian, M., Chen, Q., Zhang, Y., Du, F, Hu, H., \& Chao, F. (2014). PET imaging reveals brain functional Changes in internet gaming disorder. Vol 41, Issue7, p.1388-1397

viii. Wee, C.Y., Zhao, Z. M., Yap, P., Wu, G.R., \& Shi, F., \& Price, T. (Feb.2014). Disrupted brain functional network in internet addiction disorder: a resting state functional magnetic resonance Imagery study. S. Hayasaka (Ed). https://journals.plos.org >article>jo....

ix. Wu, C.Y., Lee, M.B., Liao, S., \& Chang, L. (Oct.2015). Risk factors in internet addiction among Internet users: an online questionnaire survey. PloS ONE 10(10): e0137506.doi: 10.1371/journal.pone.0137506 